\section{(6) OPEN ACCESS}

\title{
Impaired perfusion modifies the relationship between blood pressure and stroke risk in major cerebral artery disease
}

\author{
Hiroshi Yamauchi, Tatsuya Higashi, Shinya Kagawa, Yoshihiko Kishibe, \\ Masaaki Takahashi
}

Division of PET Imaging, Shiga Medical Centre Research Institute, Shiga, Japan

\section{Correspondence to} Dr Hiroshi Yamauchi, Division of PET Imaging, Shiga Medical Centre Research Institute, 5-430 Moriyama, Moriyama-city, Shiga 524-8524, Japan; yamauchi@res.med.shiga-pref.jp

Received 7 February 2013 Revised 24 May 2013 Accepted 10 July 2013 Published Online First 9 August 2013



To cite: Yamauchi $\mathrm{H}$, Higashi T, Kagawa $\mathrm{S}$, et al. I Neurol Neurosurg Psychiatry 2013;84: 1226-1232.

\section{ABSTRACT}

Objective Blood pressure (BP) lowering may increase stroke risk in patients with symptomatic major cerebral artery disease and impaired perfusion. To investigate the relationships among BP, impaired perfusion and stroke risk.

Methods We retrospectively analysed data from 130 non-disabled, medically treated patients with either symptomatic extracranial carotid occlusion or intracranial stenosis or occlusion of the carotid artery or middle cerebral arteries. All patients had baseline haemodynamic measurements with ${ }^{15} \mathrm{O}$-gas positron emission tomography and were followed for 2 years or until stroke recurrence or death.

Results There was a negative linear relationship between systolic BP (SBP) and risk of stroke in the territory of the diseased artery. The 2-year incidence of ischaemic stroke in the territory in patients with normal SBP (<130 mm Hg, 5/32 patients) was significantly higher than in patients with high $\operatorname{SBP}(2 / 98, p<0.005)$ Multivariate analysis revealed that normal SBP and impaired perfusion were independently associated with increased risk of stroke in the previously affected territory, while risk of stroke elsewhere was positively correlated with SBP. Overall, high total stroke risk was observed at lower BP in patients with impaired perfusion and at higher BPs in patients without (interaction, $p<0.01)$. Overall, the relationship between SBP and total stroke recurrence was J-shaped.

Conclusions Impaired perfusion modified the relationship between blood pressure and stroke risk, although this study had limitations including the retrospective analysis, the potentially biased sample, the small number of critical events and the fact that BP was measured only as a snapshot in clinic.

\section{INTRODUCTION}

In patients with atherosclerotic internal carotid artery (ICA) or middle cerebral artery (MCA) occlusive disease, chronic reduction in cerebral perfusion pressure (chronic haemodynamic compromise) increases the risk of ischaemic stroke ${ }^{1-4}$ and can be detected by directly measuring haemodynamic parameters. ${ }^{5-13}$ Accurate evaluation of haemodynamic status is essential for determining patient prognosis. Furthermore, therapeutic strategies to prevent recurrent strokes should differ between patients with and without haemodynamic compromise. However, strategies for selecting treatments based on haemodynamic measurements have not been clearly established. ${ }^{14}$

Identification of patients with impaired perfusion may be essential for informing the proper control of blood pressure (BP) to reduce stroke recurrence. Hypertension is a major risk factor for stroke, and antihypertensive therapy provides general benefits to patients with a history of stroke or transient ischaemic attacks (TIAs). ${ }^{15} 16$ However, the level to which BP should be lowered to achieve maximal benefits among survivors of stroke and TIAs is not precisely known, although post hoc analysis of PROGRESS $^{17}$ suggests a goal $<130 \mathrm{~mm} \mathrm{Hg}$ systolic BP (SBP). However, there had been concern that lowering BP might impair cerebral perfusion in patients with atherosclerotic ICA or MCA occlusive disease $^{18}$ and thereby increase stroke risk. ${ }^{2}$ Previous studies have demonstrated that the risk of stroke increases with BP in the great majority of patients with stenosis of the extracranial ICA or intracranial large artery, ${ }^{19-21}$ while patients with symptomatic bilateral carotid stenosis $>70 \%$ exhibit an inverse relationship between BP and stroke risk. ${ }^{20}$ Notably, these studies did not include direct evaluations of baseline patient haemodynamic status. They also did not include patients with symptomatic occlusion of the major cerebral arteries, in which the incidence of impaired perfusion is greater than in patients with stenosis of the major cerebral arteries. Thus, the interpretation of these studies was limited by the fact that the proportion of patients with impaired perfusion was unknown and potentially low. The relationship between BP and stroke risk in patients with impaired perfusion may be different from that in patients without impaired perfusion, and lower BP in this specific population may increase recurrent stroke risk. ${ }^{20}$ Therefore, a more thorough characterisation of the relationships among perfusion, BP and stroke risk should be performed to inform selection of the most appropriate treatment regimen for individual patients.

In this study, we retrospectively analysed the relationship between BP during follow-up and stroke risk in a population of a relatively large number of patients with symptomatic ICA or MCA occlusion. We quantitatively evaluated baseline haemodynamic status with positron emission tomography (PET) and ${ }^{15} \mathrm{O}$-gas. The purpose of this study was to investigate the relationships among BP, impaired perfusion and recurrent stroke risk in symptomatic major cerebral artery occlusive disease. 


\section{MATERIALS AND METHODS}

Patients

We retrospectively analysed the data collected from 130 medically treated patients enrolled in an observational study that investigated the relationship between haemodynamic compromise and stroke risk in symptomatic patients with atherosclerotic occlusive disease of the major cerebral arteries other than extracranial ICA stenosis. ${ }^{13}$ Patients were first referred to the PET unit at Shiga Medical Centre from the outpatient clinic or other hospitals in Shiga Prefecture to undergo haemodynamic parameter evaluation as part of clinical assessment to determine the need for extracranial-to-intracranial bypass. Although the benefit from bypass surgery in patients with haemodynamic compromise remains to be proven, the operation is nevertheless performed in some patients in Japan and elsewhere.

Inclusion criteria for the observational study were as follows: (1) occlusion of the extracranial ICA or occlusion or stenosis ( $>50 \%$ diameter reduction) of the intracranial ICA or MCA as documented by conventional or magnetic resonance angiography, (2) ability to independently carry out daily life activities (modified Rankin scale score $<3$ ) and (3) history of TIA or complete stroke involving the relevant ICA or MCA territory at any time before PET examination. TIA was defined as the development of focal symptoms of presumed ischaemic cerebrovascular origin lasting $<24 \mathrm{~h}$. The exclusion criteria were (1) history of vascular reconstruction surgery or (2) presence of potential sources of cardiogenic embolism. ${ }^{13}$ Overall, 35 of the 165 patients enrolled in the study underwent bypass surgery due to haemodynamic impairment observed on PET and were excluded from the present study.

The study included 103 men and 27 women aged 4490 years (mean \pm SD: $64 \pm 8$ years, table 1 ). Twenty-one patients had TIA, and 109 had minor stroke. All but two patients had symptoms of cerebral hemispheric (rather than retinal) ischaemia. The interval between the latest ischaemic event and PET evaluation was $8 \pm 15$ months (range, 4 days to 72 months). Recurrent symptoms prior to PETwere identified in 52 patients, including 25 after angiographic demonstration of arterial disease. In 9 of 21 patients with TIA, MRI was normal. In 121 patients, MRI revealed only minor abnormalities in the MCA territory or watershed areas of the hemisphere with symptomatic arterial disease. The symptomatic qualifying artery included the extracranial ICA occlusion in 61 cases, intracranial ICA occlusion in 1 case, intracranial ICA stenosis in 13 cases, MCA occlusion in 24 cases and MCA stenosis in 31 cases. Among vascular risk factors, hypertension, diabetes mellitus, ischaemic heart disease, hypercholesterolaemia and smoking status were evaluated from patient histories recorded at PET examination (table 1). Hypertension, diabetes mellitus, ischaemic heart disease and hypercholesterolaemia were judged as present based on treatment history. The ethics committee of our centre approved the study protocol, and each patient provided written informed consent prior to their participation in this study.

Table 1 Characteristics of patients with and without normal SBP $(<130 \mathrm{~mm} \mathrm{Hg})$ during follow-up

\begin{tabular}{|c|c|c|c|}
\hline \multirow[b]{3}{*}{ Characteristics } & \multirow[b]{3}{*}{ Total } & \multicolumn{2}{|c|}{ Categorisations } \\
\hline & & \multicolumn{2}{|l|}{ Normal SBP } \\
\hline & & Present & Absent \\
\hline Number of patients & 130 & 32 & 98 \\
\hline Age, mean $\pm S D, y$ & $64 \pm 8$ & $63 \pm 10$ & $64 \pm 7$ \\
\hline Sex, male/female, number & $103 / 27$ & $26 / 6$ & $77 / 21$ \\
\hline \multicolumn{4}{|l|}{ Diagnosis, number } \\
\hline TIA (amaurosis/hemispheric) & $21(2 / 19)$ & $6(0 / 6)$ & $15(2 / 13)$ \\
\hline Minor stroke & 109 & 26 & 83 \\
\hline Recurrent symptoms, number & 52 & 16 & 36 \\
\hline After demonstration of arterial disease & 25 & 8 & 17 \\
\hline Number of months between last symptom and PET, mean \pm SD & $8 \pm 15$ & $9 \pm 16$ & $8 \pm 15$ \\
\hline Recent (6 $\mathrm{m}$ or less) & 100 & 25 & 75 \\
\hline \multicolumn{4}{|l|}{ Symptomatic qualifying artery, number } \\
\hline Occlusion/stenosis & $86 / 44$ & $21 / 11$ & $65 / 33$ \\
\hline Extracranial ICA occlusion & 61 & 15 & 46 \\
\hline Intracranial ICA (occlusion/stenosis) & $14(1 / 13)$ & $3(1 / 2)$ & $11(0 / 11)$ \\
\hline MCA (occlusion/stenosis) & $55(24 / 31)$ & $14(5 / 9)$ & $41(19 / 22)$ \\
\hline \multicolumn{4}{|l|}{ Other medical illness, number } \\
\hline Hypertension & 78 & 20 & 58 \\
\hline $\mathrm{Ca}^{2+}$-antagonist & 43 & 9 & 34 \\
\hline ACE inhibitor & 17 & 5 & 12 \\
\hline ARB & 18 & 6 & 12 \\
\hline Diabetes mellitus & 47 & 9 & 38 \\
\hline Ischaemic heart disease & 27 & 10 & 17 \\
\hline Hypercholesterolaemia & 40 & 9 & 31 \\
\hline Smoking habit (current and former), number & 46 & 14 & 32 \\
\hline Decreased CBF/CBV & 39 & 13 & 26 \\
\hline Blood pressure during follow-up (mm Hg) (systolic/diastolic) & $142 \pm 19 / 72 \pm 10$ & $119 \pm 7 / 66 \pm 9$ & $149 \pm 16 / 74 \pm 9$ \\
\hline
\end{tabular}

ACE, angiotensin-converting enzyme; $A R B$, angiotensin receptor blocker; $C B F$, cerebral blood flow; CBV, cerebral blood volume; ICA, internal carotid artery; MCA, middle cerebral artery; PET, positron emission tomography; SBP, systolic blood pressure; TIA, transient ischaemic attack. 


\section{PET measurements}

All patients underwent PET scans with a whole-body Advance PET scanner (General Electric Medical System, Wauwatosa, WI), which permits simultaneous acquisition of 35 image slices with interslice spacing of $4.25 \mathrm{~mm} .{ }^{22}$ The intrinsic scanner resolution was $4.6-5.7 \mathrm{~mm}$ in the transaxial direction and $4.0-5.3 \mathrm{~mm}$ in the axial direction. As part of the scanning procedure but before tracer administration, ${ }^{68} \mathrm{Ge} /{ }^{68} \mathrm{Ga}$ transmission scanning was performed for $10 \mathrm{~min}$ for attenuation correction. Functional images were reconstructed as $128 \times 128$ pixels, with each pixel representing an area of $2.0 \times 2.0 \mathrm{~mm}$.

A series of ${ }^{15} \mathrm{O}$-gas studies was also performed. $\mathrm{C}^{15} \mathrm{O}_{2}$ and ${ }^{15} \mathrm{O}_{2}$ were inhaled continuously through a mask. ${ }^{22}$ The scan time was $5 \mathrm{~min}$. Bolus inhalation of $\mathrm{C}^{15} \mathrm{O}$ with 3 -min scanning was used to measure cerebral blood volume (CBV). Arterial samples were manually obtained during scanning.

Cerebral blood flow (CBF), cerebral metabolic rate of oxygen and oxygen extraction fraction (OEF) were calculated based on the steady-state method. ${ }^{23}$ The ratio of CBF to CBV was calculated pixel-by-pixel as an indicator of cerebral perfusion pressure. $^{2526}$

\section{Data analysis}

We analysed 10 tomographic planes from 46.25 to $84.5 \mathrm{~mm}$ above and parallel to the orbitomeatal line, which corresponded to the levels from the basal ganglia and thalamus to the centrum semiovale. The region of interest (ROI) was placed on the CBF images. Each image was examined by placing 10-12 circular ROIs $16 \mathrm{~mm}$ in diameter compactly over the grey matter of the outer cortex in each hemisphere. According to the atlas, ${ }^{27}$ the ROIs in all 10 images covered the distribution of the MCA, as well as the watershed areas. ${ }^{28}$ The same ROIs were transferred to the other images. The mean hemispheric values in each hemisphere were calculated as the average of the values of all circular ROIs. In patients with cerebral cortex infarction, the circular ROIs that overlapped low-intensity areas on T1-weighted MR images were excluded from analysis using a simple method correlating PET images with MR images. ${ }^{29}$

Normal control values of the ${ }^{15} \mathrm{O}$-gas PET variables were obtained from seven normal volunteers (four men and three women), aged $47 \pm 7$ years (mean $\pm S D$ ) who underwent normal routine neurological examinations and MRI scans. The mean OEF value obtained from these 14 control hemispheres was $44.5 \%$ $\pm 3.8 \%$. Hemispheric OEF values beyond the upper 95\% limit defined in normal subjects (above 52.9\%) were considered to represent increased OEF. Comparative values for $\mathrm{CBF}$ and $\mathrm{CBF} / \mathrm{CBV}$ in normal controls were $44.6 \pm 4.5$ and $11.4 \pm 1.8$, respectively. Hemispheric CBF and CBF/CBV values below $35.0 \mathrm{~mL} / 100 \mathrm{~g} / \mathrm{min}$ and $7.6 / \mathrm{min}$, respectively, were considered abnormal.

Patients with increased OEF, decreased CBF and decreased $\mathrm{CBF} / \mathrm{CBV}$ in hemispheres with arterial disease were categorised as having misery perfusion, while patients with decreased $\mathrm{CBF} / \mathrm{CBV}$ were categorised as having decreased cerebral perfusion pressure (impaired perfusion). Patients were categorised by an investigator who was unaware of their clinical status.

\section{Follow-up and outcome}

Attending physicians were informed of PET findings, but treatment of risk factors and use of drugs was left to individual clinical judgment. All patients were examined at 1-month or 2-month intervals after PET studies in the outpatient clinic in our centre or related hospitals in Shiga Prefecture. At each visit, an interim history was obtained, BP was measured and a neurological examination was performed. In patients with recurrent stroke, MR imaging or CT scan was obtained and compared with initial studies to confirm recurrent stroke. Patients were followed for 2 years or until stroke recurrence or death. Recurrent ischaemic stroke was defined as the acute onset of new focal neurological deficit of cerebral origin persisting for more than $24 \mathrm{~h}$ without primary intracranial haemorrhage on CT or MRI scan.

$\mathrm{BP}$ during the follow-up period was defined as the value of BP obtained just before stroke recurrence, death or the end of the 2-year follow-up period. Continuous SBP measurements were categorised in the same way as in the study by Rothwell et al $(<130$, 130-149, 150-169 and $\geq 170 \mathrm{~mm} \mathrm{Hg}$ ). ${ }^{20}$ We defined normal BP as $\mathrm{SBP}<130 \mathrm{~mm} \mathrm{Hg}$ or diastolic BP (DBP) $<85 \mathrm{~mm} \mathrm{Hg}^{30} 31$ For persons with higher values (SBP $130-139 \mathrm{~mm} \mathrm{Hg}$ or DBP $85-89 \mathrm{~mm} \mathrm{Hg}$ ) within the prehypertensive range, stroke risk is reported to be substantially increased. ${ }^{32}$ A guideline recommends treatment for subjects with SBP $\geq 130 \mathrm{~mm} \mathrm{Hg}^{31}$

\section{Statistical analysis}

Clinical backgrounds were compared between groups using Student $t$ tests or $\chi^{2}$ tests, as appropriate; significance was established at $\mathrm{p}<0.05$. The incidence of recurrent stroke was compared between groups using Mantel-Cox log-rank statistics and Kaplan-Meier survival curves. Survival analysis of subsequent endpoints began on the day of PET examination, which was considered the date of entry into the study. Multivariate analysis with the Cox proportional hazards model was used to test the effect of multiple variables on stroke recurrence. Covariate selection was performed by including the following covariates in a stepwise model: age, sex, recurrent symptoms (recurrent episodes of ischaemic attack prior to PET or after angiographic demonstration of arterial disease), time between the last symptoms and PET, symptomatic arterial occlusion, extracranial ICA occlusion, complications (hypertension, diabetes mellitus, prior ischaemic heart disease, hypercholesterolaemia), smoking habit, BP during follow-up (categorisation or normal BP) and decreased $\mathrm{CBF} / \mathrm{CBV}$ or misery perfusion. A forward stepwise selection was performed, and covariates were included and selected based on a significant relationship $(p<0.05)$ with an outcome event to enter into the model and $\mathrm{p}<0.05$ to remain in the model. The selected covariates were then included in a final model for analysis. The difference of the relationship of follow-up BP level with recurrent strokes in different subgroups of participants (patients with or without impaired perfusion) was evaluated by adding an interaction term to the model.

\section{RESULTS}

Thirty-two of the 130 patients had normal SBP $(<130 \mathrm{~mm} \mathrm{Hg})$ during follow-up (table 1). These same patients also had normal DBP ( $<85 \mathrm{~mm} \mathrm{Hg}$ ) except for one patient $(88 \mathrm{~mm} \mathrm{Hg})$. None of the patient characteristics were significantly different between patients with normal SBP and those without. All but two patients in our study were treated with antiplatelet therapy.

On the basis of the CBF/CBV values in the hemisphere supplied by the previously symptomatic artery, 39 patients $(30.0 \%)$ had decreased cerebral perfusion pressure, and 91 (70.0\%) did not. Sixteen of the 39 patients with decreased CBF/CBV had misery perfusion. The incidence of decreased $\mathrm{CBF} / \mathrm{CBV}$ in patients with arterial occlusion $(34 / 86,39.5 \%)$ was significantly higher than that in patients with stenosis $(5 / 44,11.3 \%)(p=0.001)$.

There were seven ischaemic strokes in the territory of the diseased artery, and six (including two haemorrhages) in other vascular territories. One of the 13 strokes was fatal (haemorrhage). 
None of the patients was lost to follow-up. In univariate analysis using the Cox proportional hazards model (table 2), there was a negative linear relationship between SBP and risk of stroke in the territory of the diseased artery; the relative risk per $20 \mathrm{~mm} \mathrm{Hg}$ was 0.27 (95\% CI 0.08 to $0.93, \mathrm{p}<0.05)$. On the other hand, there was a positive linear relationship between SBP and risk of stroke in the other vascular territory; the relative risk per $20 \mathrm{~mm} \mathrm{Hg}$ was 4.02 (95\% CI 1.4 to $10.8, \mathrm{p}<0.01)$. The 2 -year incidence of ischaemic stroke in the territory of the diseased artery in patients with normal SBP $(5 / 32 ; 15.6 \%)$ was significantly higher than that in patients without normal SBP (2/ $98,2.0 \%, \mathrm{p}<0.005$; figure 1 , table 3 ). We observed no new haemorrhagic strokes in the previously affected territory. All five patients with normal SBP and recurrent stroke had been taking BP medication. Six strokes, including two haemorrhages, in the other vascular territory occurred only in patients without normal SBP (6.1\%). Therefore, the total stroke incidence was 5/ $32(15.6 \%)$ in patients with normal SBP and 8/98 (8.2\%) in patients without. Four patients without normal SBP died of non-cerebral causes during follow-up.

In multivariate analysis using the Cox proportional hazards model, normal SBP and decreased CBF/CBV were selected and included in the final model for stroke in the territory of the diseased artery. Normal SBP and decreased CBF/CBV were independently associated with increased risk of ischaemic stroke in the territory. The relative risk conferred by the presence of normal SBP and decreased CBF/CBV was 6.3 (95\% CI 1.2 to 32.6, $\mathrm{p}<0.05)$ and 12.3 (95\% CI 1.5 to $102.9, \mathrm{p}<0.05)$, respectively. When misery perfusion was used as a covariate instead of decreased $\mathrm{CBF} / \mathrm{CBV}$, only misery perfusion was selected and included in the final model. For stroke in the other vascular territory, only SBP (categorisation) was selected and included in the final model. The risk of stroke in the other vascular territory was positively correlated with SBP.

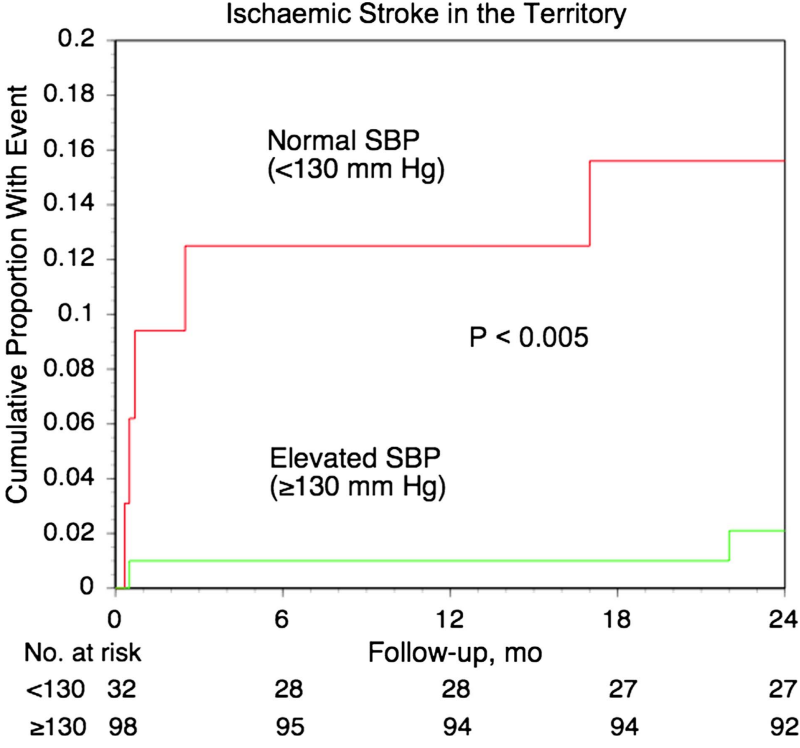

Figure 1 Kaplan-Meier cumulative failure curves for ipsilateral ischaemic stroke in patients with and without normal SBP. The number of patients who remained event-free and available for follow-up evaluation during each 6-month interval is shown at the bottom of the graph. SBP, systolic blood pressure.

In the subgroup comparisons of total stroke recurrence rate with SBP, high total stroke risk was observed at lower BP values in patients with impaired perfusion and at higher BP values in patients without (interaction, $\mathrm{p}<0.01$; figure 2 ). When data on both subgroups were combined, the 2-year incidence of total strokes in patients with values of SBP within the 130 $149 \mathrm{~mm} \mathrm{Hg}$ range $(2 / 57,3.6 \%)$ was significantly lower than in those whose BP fell outside of that range (11/73, 15.1\%,

Table 2 Univariate analysis of recurrent stroke risk factors

\begin{tabular}{|c|c|c|c|c|c|c|}
\hline & \multicolumn{2}{|c|}{ Arterial territory } & \multirow[b]{2}{*}{ HR $(95 \% \mathrm{Cl})$} & \multicolumn{2}{|c|}{ Other territory } & \multirow[b]{2}{*}{ HR $(95 \% \mathrm{CI})$} \\
\hline & Yes & No & & Yes & No & \\
\hline Number of patients & 7 & 123 & & 6 & 124 & \\
\hline Age, mean $\pm S D$, y & $66 \pm 5$ & $64 \pm 8$ & $1.03(0.94$ to 1.12$)$ & $68 \pm 5$ & $64 \pm 8$ & $1.07(0.97$ to 1.17$)$ \\
\hline Sex, male, number (\%) & $6(86)$ & $97(79)$ & $1.6(0.2$ to 13.8$)$ & $3(50)$ & $100(81)$ & $0.26(0.05$ to 1.32$)$ \\
\hline Recurrent symptoms & $4(57)$ & $48(39)$ & $2.0(0.4$ to 9.0$)$ & $2(33)$ & $50(40)$ & $0.78(0.14$ to 4.3$)$ \\
\hline After demonstration of arterial disease & $2(29)$ & $23(19)$ & $1.7(0.3$ to 8.9$)$ & $1(17)$ & $24(19)$ & $0.88(0.1$ to 7.5$)$ \\
\hline Number of months between last symptom and PET, mean \pm SD & $18 \pm 23$ & $8 \pm 15$ & 1.02 (0.99 to 1.05$)$ & $3 \pm 4$ & $8 \pm 16$ & 0.96 (0.85 to 1.07$)$ \\
\hline Recent (6 m or less) & $4(57)$ & $96(78)$ & 0.38 (0.08 to 1.73$)$ & $5(83)$ & $95(77)$ & 1.43 (0.16 to 12.2$)$ \\
\hline Arterial occlusion & $3(43)$ & $83(67)$ & $0.38(0.08$ to 1.70$)$ & $3(50)$ & $83(67)$ & $0.48(0.09$ to 2.39$)$ \\
\hline Extracranial ICA occlusion & $3(43)$ & $58(47)$ & $0.86(0.19$ to 3.85$)$ & $3(50)$ & $58(47)$ & $1.14(0.23$ to 5.67$)$ \\
\hline Hypertension & $6(86)$ & $72(59)$ & $4.1(0.49$ to 34.2$)$ & $4(67)$ & $74(60)$ & $1.4(0.26$ to 7.7$)$ \\
\hline Diabetes mellitus & $3(43)$ & $44(36)$ & $1.3(0.3$ to 6.0$)$ & $3(50)$ & $44(35)$ & $1.8(0.1$ to 7.3$)$ \\
\hline Ischaemic heart disease & $3(43)$ & $24(20)$ & $3.0(0.6$ to 13.7$)$ & $1(17)$ & $26(21)$ & $0.8(0.6$ to 13.7$)$ \\
\hline Hypercholesterolaemia & $2(29)$ & $38(31)$ & $0.87(0.16$ to 4.5$)$ & $2(33)$ & $38(31)$ & 1.07 (0.19 to 5.8$)$ \\
\hline Smoking (current and former) & $4(57)$ & $42(34)$ & $2.4(0.54$ to 10.8$)$ & $2(33)$ & $44(35)$ & $0.9(0.16$ to 4.9$)$ \\
\hline \multicolumn{7}{|l|}{ SBP } \\
\hline Categorisation & $1.4 \pm 0.7$ & $2.2 \pm 0.8$ & $0.27(0.08$ to 0.93$)$ & $3.3 \pm 0.8$ & $2.1 \pm 0.8$ & $4.02(1.4$ to 10.8$)$ \\
\hline Normal BP & $5(71)$ & $27(22)$ & 8.1 (1.5 to 41.8$)$ & $0(0)$ & $26(21)$ & NE \\
\hline Decreased CBF/CBV & $6(86)$ & $33(27)$ & 15.0 (1.8 to 125.0$)$ & $1(17)$ & $38(31)$ & 0.5 (0.06 to 4.4$)$ \\
\hline Misery perfusion & $4(57)$ & $12(10)$ & $10.1(2.2$ to 45.2$)$ & $0(0)$ & $16(13)$ & NE \\
\hline
\end{tabular}

Categorisation=1, <130; 2, 130-149; 3, 150-169; 4, $\geq 170 \mathrm{~mm} \mathrm{Hg}$.

$\mathrm{BP}$, blood pressure; CBF, cerebral blood flow; CBV, cerebral blood volume; ICA, internal carotid artery; NE, not estimable; PET, positron emission tomography; SBP, systolic blood pressure. 
Table 3 Two-year stroke occurrence and SBP

\begin{tabular}{|c|c|c|c|c|}
\hline & \multicolumn{4}{|l|}{$\mathrm{SBP}, \mathrm{mm} \mathrm{Hg}$} \\
\hline & $<130$ & $130-149$ & $150-169$ & $>170$ \\
\hline \multicolumn{5}{|l|}{ Ischaemic stroke in territory } \\
\hline Total $(n=130)$ & $5 / 32(15.6 \%)$ & $1 / 57(1.8 \%)$ & $1 / 29(3.4 \%)$ & $0 / 12(0 \%)$ \\
\hline With misery perfusion* $(n=16)$ & $3 / 8(37.5 \%)$ & $0 / 3(0 \%)$ & $1 / 4(25 \%)$ & $0 / 1(0 \%)$ \\
\hline Without misery perfusion $(n=114)$ & $2 / 24(8.3 \%)$ & $1 / 54(1.9 \%)$ & $0 / 25(0 \%)$ & $0 / 11(0 \%)$ \\
\hline Decreased $C B F / C B V^{*}(n=39)$ & $4 / 13(30.8 \%)$ & $1 / 15(6.7 \%)$ & $1 / 10(10 \%)$ & $0 / 16(0 \%)$ \\
\hline Normal CBF/CBV $(n=91)$ & $1 / 19(5.3 \%)$ & $0 / 42(0 \%)$ & $0 / 19(0 \%)$ & $0 / 11(0 \%)$ \\
\hline \multicolumn{5}{|l|}{ Stroke in other vascular territory } \\
\hline Total $(n=130)$ & $0 / 32(0 \%)$ & $1 / 57(1.8 \%)$ & $2 / 29(6.9 \%)$ & $3 / 12(25 \%)$ \\
\hline With misery perfusion* $(n=16)$ & $0 / 8(0 \%)$ & $0 / 3(0 \%)$ & $0 / 4(0 \%)$ & $0 / 1(0 \%)$ \\
\hline Without misery perfusion $(n=114)$ & $0 / 24(0 \%)$ & $1 / 54(1.9 \%)$ & $2 / 25(8 \%)$ & $3 / 11(27.3 \%)$ \\
\hline Decreased CBF/CBV* $(n=39)$ & $0 / 13(0 \%)$ & $1 / 15(6.7 \%)$ & $0 / 10(0 \%)$ & $0 / 16(0 \%)$ \\
\hline Normal CBF/CBV $(n=91)$ & $0 / 19(0 \%)$ & $0 / 42(0 \%)$ & $2 / 19(10.5 \%)$ & $3 / 11(27.3 \%)$ \\
\hline
\end{tabular}

$\mathrm{p}<0.05$; figure 2). Overall, the relationship between SBP and total stroke recurrence was J-shaped.

When DBP values were categorised as $<75,75-84$ and $\geq 85 \mathrm{~mm} \mathrm{Hg}$, a similar tendency was observed between DBP and stroke risk without statistical significance; the 2-year incidence of total strokes in each category was 5/27 (19\%), 2/8 (25\%) and $0 / 4(0 \%)$ in patients with impaired perfusion, and 4/43 (9.3\%), $0 / 36(0 \%)$ and $2 / 12(17 \%)$ in patients without, respectively.

\section{DISCUSSION}

This study demonstrated that lower SBP during follow-up and impaired perfusion significantly increased the risk of ischaemic stroke in the territory of the diseased artery, while higher BP significantly increased the risk of stroke in other vascular territories. We demonstrated this by following 130 patients with symptomatic major cerebral arterial occlusive disease, including 86 patients with arterial occlusion. Overall, high total stroke risk was observed at lower BP values in patients with impaired perfusion and at higher BP values in patients without.

\section{Control of BP based on haemodynamic measurements}

Our results indicate that the relationship between follow-up BP level and recurrent stroke risk differs between patients with or



Figure 2 Line graph showing subgroup comparisons of total stroke recurrence rate as a function of systolic blood pressure. without impaired perfusion. We observed a statistically significant interaction between the presence of impaired perfusion and the SBP-stroke risk relationship in the present study. This finding supports a previous report documenting a similar interaction between the presence of bilateral severe ICA stenosis and the SBP-stroke risk relationship that may have been influenced by the presence of impaired perfusion. ${ }^{20}$ Impaired perfusion is probably absent in the majority of patients with unilateral stenosis of the extracranial ICA or intracranial large artery, which may explain the reported increase in stroke risk with BP in such patients. ${ }^{19-21}$ Lower BP during follow-up may increase the risk of recurrent stroke in patients with impaired perfusion at baseline.

Aggressive medical managements for secondary prevention may progressively decrease recurrent stroke risk in patients with ICA or MCA occlusive disease in general. ${ }^{33}{ }^{34}$ Treatment of hypertension may prevent progression of large or small cerebral artery disease, which may decrease stroke recurrence. BP control is also needed to prevent cerebral haemorrhage during antiplatelet therapy. However, the present results suggest that patients with impaired perfusion should be treated differently to those without. BP could be strictly controlled only in patients without impaired perfusion, but aggressive BP lowering should be avoided in patients with impaired perfusion. In the present study, patients with impaired perfusion who had normal BP $(<130 \mathrm{~mm} \mathrm{Hg})$ and recurrent stroke during a follow-up visit exhibited higher BP $(\geq 130 \mathrm{~mm} \mathrm{Hg})$ at baseline, while no patients with impaired perfusion who exhibited normal BP at baseline and normal or higher BP during follow-up had recurrent stroke (data not shown). Thus, BP lowering per se, as well as a lower target BP, might be associated with stroke risk in patients with impaired perfusion. We do not recommend lowering BP to the normal level $(<130 \mathrm{~mm} \mathrm{Hg})$ in patients who had impaired perfusion and higher $\mathrm{BP}(\geq 130 \mathrm{~mm} \mathrm{Hg})$ at baseline. Treatment with antihypertensive drugs may be safe and reduce stroke recurrence only when individual haemodynamic status is correctly evaluated and an individualised goal is selected based on the presence of impaired perfusion.

\section{J-shaped association}

The presence of a J-shaped association between BP and risk of recurrent stroke is a matter of debate. ${ }^{17} 35$ In the present study, the 
relationship between SBP and total stroke recurrence was J-shaped. The incidence of total strokes was lowest in patients with SBP values within the $130-149 \mathrm{~mm} \mathrm{Hg}$ range. Furthermore, the present results clearly demonstrate that this J-shaped association was caused by the combined effects of the negative association with recurrent stroke in patients with impaired perfusion and the positive association with recurrent stroke in patients without impaired perfusion. The negative association in patients with impaired perfusion would be masked in a patient sample with a low prevalence of impaired perfusion. ${ }^{20}$ The difference of the proportion of patients with impaired perfusion and large artery disease may have contributed to the discrepant results among previous studies investigating the association between BP and stroke recurrence. ${ }^{17} 35$

\section{Limitations}

This study was a post hoc analysis of an observational study based on just 13 strokes in 130 selected patients; therefore, we cannot exclude the possibility that unmeasured confounding variables may explain some of our findings. The patients had a mixture of ICA and MCA diseases, which may make it difficult to accurately compare patient groups. In addition to referral bias, exclusion of patients who underwent bypass may have caused selection bias. Although patients with bypass surgery had a higher incidence of impaired perfusion, they had similar baseline clinical characteristics. BP was obtained with a single-visit measurement, which may have led to a misclassification of BP levels. Although the BP measurements might lead to a change in treatment, using BP measured at the last visit might allow analysis of the relationship between stroke and BP near the time of the event. In some patients, haemodynamic compromise severity might not have been reflected by PET variables in the whole hemisphere; rather, they may have been more regional (eg, in a watershed region). However, such patients with regional haemodynamic compromise may be less susceptible to BP decreases.

Correct evaluations of haemodynamic status using ${ }^{15} \mathrm{O}$-gas PET could identify a subgroup of patients with impaired perfusion and high stroke risk at lower BPs. The CBF/CBV is the reciprocal of the expression for vascular mean transit time (MTT) that can be evaluated by perfusion imaging with MRI or CT in routine clinical practice. $^{36} 37$ Hemispheric CBF/CBV values below $7.6 / \mathrm{min}$ correspond to MTT values above $7.8 \mathrm{~s}$. Future studies should use perfusion MRI or CT methods to assess relationships among impaired perfusion, BP and stroke recurrence in a larger patient sample. A randomised controlled trial including direct haemodynamic measurement is needed to determine the level to which BP should be lowered to achieve maximal benefits in patients with or without impaired perfusion.

\section{CONCLUSIONS}

In patients with symptomatic major cerebral arterial occlusive disease, impaired perfusion modifies the relationship between $\mathrm{BP}$ and stroke risk. Total stroke risk may be high at lower BP in patients with impaired perfusion and at higher BP in patients without. For appropriate BP control, patients with and without impaired perfusion should be treated differently, and aggressive BP lowering should be avoided in patients with impaired perfusion. Thus, the identification of patients with impaired perfusion is essential for regulating BP to reduce stroke recurrence.

Acknowledgements We thank the staff of the Division of PET Imaging, Shiga Medical Centre Research Institute for their support and technical help. We thank the staff of the Departments of Neurology and Neurosurgery of Shiga Medical Centre, Japanese Red Cross Otsu Hospital, Shiga University of Medical Science Hospital, Saiseikai Shigaken Hospital, Kusatsu General Hospital, Yasu Hospital, Omi Hachiman
City Hospital, Nagahama Red Cross Hospital and Takashima General Hospital for providing clinical assistance.

Contributors HY was responsible for the design of the study. All authors were responsible for data extraction, and TH and HY performed the analysis. HY was involved in drafting the manuscript. All authors verified the analysis and were involved in the critical revision of the manuscript. HY is the guarantor.

Funding This study was supported in part by a Grant-in-Aid for Scientific Research from the Japan Society for the Promotion of Science (22613001).

\section{Competing interests None.}

Ethics approval The Ethics Committee of Shiga Medical Centre.

Provenance and peer review Not commissioned; externally peer reviewed.

Open Access This is an Open Access article distributed in accordance with the Creative Commons Attribution Non Commercial (CC BY-NC 3.0) license, which permits others to distribute, remix, adapt, build upon this work non-commercially, and license their derivative works on different terms, provided the original work is properly cited and the use is non-commercial. See: http://creativecommons.org/ licenses/by-nc/3.0/

\section{REFERENCES}

1 Baron JC, Bousser MG, Rey A, et al. Reversal of focal "misery-perfusion syndrome" by extra-intracranial arterial bypass in hemodynamic cerebral ischemia. A case study with 150 positron emission tomography. Stroke 1981;12:454-9.

2 Klijn CJ, Kappelle LJ. Haemodynamic stroke: clinical features, prognosis, and management. Lancet Neurol 2010;9:1008-17.

3 Powers WJ. Cerebral hemodynamics in ischemic cerebrovascular disease. Ann Neurol 1991;29:231-40

4 Yamauchi $\mathrm{H}$, Fukuyama $\mathrm{H}$, Fujimoto $\mathrm{N}$, et al. Significance of low perfusion with increased oxygen extraction fraction in a case of internal carotid artery stenosis. Stroke 1992;23:431-2.

5 Derdeyn CP, Grubb RL Jr, Powers WJ. Cerebral hemodynamic impairment: methods of measurement and association with stroke risk. Neurology 1999;53:251-9.

6 Grubb RL Jr, Derdeyn CP, Fritsch SM, et al. Importance of hemodynamic factors in the prognosis of symptomatic carotid occlusion. JAMA 1998;280:1055-60.

7 Kuroda S, Houkin K, Kamiyama $\mathrm{H}$, et al. Long-term prognosis of medically treated patients with internal carotid or middle cerebral artery occlusion: can acetazolamide test predict it? Stroke 2001;32:2110-16.

8 Markus H, Cullinane M. Severely impaired cerebrovascular reactivity predicts stroke and TIA risk in patients with carotid artery stenosis and occlusion. Brain 2001; 124:457-67.

9 Ogasawara K, Ogawa A, Yoshimoto T. Cerebrovascular reactivity to acetazolamide and outcome in patients with symptomatic internal carotid or middle cerebral artery occlusion: a xenon-133 single-photon emission computed tomography study. Stroke 2002;33:1857-62.

10 Vernieri F, Pasqualetti P, Passarelli F, et al. Outcome of carotid artery occlusion is predicted by cerebrovascular reactivity. Stroke 1999;30:593-8.

11 Yamauchi $H$, Fukuyama $H$, Nagahama $Y$, et al. Evidence of misery perfusion and risk for recurrent stroke in major cerebral arterial occlusive diseases from PET. J Neurol Neurosurg Psychiatry 1996;61:18-25.

12 Yamauchi $H$, Fukuyama $H$, Nagahama $Y$, et al. Significance of increased oxygen extraction fraction in 5-year prognosis of major cerebral arterial occlusive diseases. J Nucl Med 1999;40:1992-8.

13 Yamauchi $\mathrm{H}$, Higashi $T$, Kagawa $\mathrm{S}$, et al. Is misery perfusion still a predictor of stroke in symptomatic major cerebral artery disease? Brain 2012;135:2515-26.

14 Powers WJ. Stroke: Misery perfusion in cerebrovascular disease-is it important? Nat Rev Neurol 2012;8:479-80.

15 PROGRESS Collaborative Study Group. Randomised trial of a perindopril-based blood-pressure-lowering regimen among 6,105 individuals with previous stroke or transient ischaemic attack. Lancet 2001;358:1033-41.

16 Rashid P, Leonardi-Bee J, Bath P. Blood pressure reduction and secondary prevention of stroke and other vascular events: a systematic review. Stroke 2003;34:2741-8.

17 Arima H, Chalmers J, Woodward M, et al. Lower target blood pressures are safe and effective for the prevention of recurrent stroke: the PROGRESS trial. $J$ Hypertens 2006;24:1201-8

18 Nagahama Y, Fukuyama $H$, Yamauchi $H$, et al. Effect of nicardipine on cerebral blood flow in hypertensive patients with internal carotid artery occlusion: a PET study. J Stroke Cerebrovasc Dis 1997;6:325-31.

19 King A, Shipley M, Markus $H$. The effect of medical treatments on stroke risk in asymptomatic carotid stenosis. Stroke 2013;44:542-6.

20 Rothwell PM, Howard SC, Spence JD. Relationship between blood pressure and stroke risk in patients with symptomatic carotid occlusive disease. Stroke 2003:34:2583-90.

21 Turan TN, Cotsonis G, Lynn MJ, et al. Relationship between blood pressure and stroke recurrence in patients with intracranial arterial stenosis. Circulation 2007:115:2969-75. 
22 Okazawa $\mathrm{H}$, Yamauchi $\mathrm{H}$, Sugimoto $\mathrm{K}$, et al. Quantitative comparison of the bolus and steady-state methods for measurement of cerebral perfusion and oxygen metabolism: Positron emission tomography study using ${ }^{15} 0$ gas and water. J Cereb Blood Flow Metab 2001;21:793-803.

23 Frackowiak RSJ, Lenzi GL, Jones T, et al. Quantitative measurement of regional cerebral blood flow and oxygen metabolism in man using ${ }^{15} \mathrm{O}$ and positron emission tomography: theory, procedure, and normal values. J Comput Assist Tomogr 1980;4:727-36.

24 Lammertsma AA, Jones T. Correction for the presence of intravascular oxygen-15 in the steady-state technique for measuring regional oxygen extraction ratio in the brain: 1. Description of the method. J Cereb Blood Flow Metab 1983;3:416-24.

25 Gibbs JM, Wise RJS, Leenders KL, et al. Evaluation of cerebral perfusion reserve in patients with carotid artery occlusion. Lancet 1984;8372:310-14.

26 Schumann P, Touzani O, Young AR, et al. Evaluation of the ratio of cerebral blood flow to cerebral blood volume as an index of local cerebral perfusion pressure. Brain 1998;121:1369-79.

27 Kretschmann HJ, Weinrich W. Neuroanatomy and Cranial Computed Tomography. New York: Thieme Inc., 1986:70-4.

28 Yamauchi $\mathrm{H}$, Fukuyama $\mathrm{H}$, Kimura J, et al. Hemodynamics in internal carotid artery occlusion examined by positron emission tomography. Stroke 1990;21:1400-6.

29 Yamauchi $\mathrm{H}$, Fukuyama $\mathrm{H}$, Harada $\mathrm{K}$, et al. White matter hyperintensities may correspond to areas of increased blood volume: correlative MR and PET observations. J Comput Assist Tomogr 1990;14:905-8.
30 Mancia G, De Backer G, Dominiczak A, et al. 2007 Guidelines for the management of arterial hypertension: The Task Force for the Management of Arterial Hypertension of the European Society of Hypertension (ESH) and of the European Society of Cardiology (ESC). Eur Heart J 2007;28:1462-536.

31 Ogihara T, Kikuchi K, Matsuoka H, et al. The Japanese Society of Hypertension Guidelines for the Management of Hypertension (JSH 2009). Hypertens Res 2009;32:3-107.

32 Lee $M$, Saver JL, Chang B, et al. Presence of baseline prehypertension and risk of incident stroke: a meta-analysis. Neurology 2011;77:1330-7.

33 Abbott AL. Medical (nonsurgical) intervention alone is now best for prevention of stroke associated with asymptomatic severe carotid stenosis: results of a systematic review and analysis. Stroke 2009;40:e573-83.

34 Chimowitz M, Lynn M, Derdeyn C, et al. Stenting versus aggressive medical therapy for intracranial arterial stenosis. N Engl J Med 2011:365:993-1003.

35 Irie K, Yamaguchi T, Minematsu K, et al. The J-curve phenomenon in stroke recurrence. Stroke 1993:24:1844-9.

36 Carrera $\mathrm{E}$, Jones PS, Iglesias $\mathrm{S}$, et al. The vascular mean transit time: a surrogate for the penumbra flow threshold? J Cereb Blood Flow Metab 2011;31:1027-35.

37 Kajimoto K, Moriwaki H, Yamada N, et al. Cerebral hemodynamic evaluation using perfusion-weighted magnetic resonance imaging: comparison with positron emission tomography values in chronic occlusive carotid disease. Stroke 2003:34:1662-6. 


\section{Correction}

H Yamauchi, T Higashi, S Kagawa et al. Impaired perfusion modifies the relationship between blood pressure and stroke risk in major cerebral artery disease. J Neurol Neurosurg Psychiatry 2013;84: 1226-1232. In table 3 of this article the number of the patients with decreased $\mathrm{CBF} / \mathrm{CBV}$ and $\mathrm{SBP}>170$ should be 1 and not 16.

J Neurol Neurosurg Psychiatry 2014;85:118. doi:10.1136/jnnp-2013-305159corr1 